\title{
Efficient emulator for solving three-nucleon continuum Faddeev equations with chiral three-nucleon force comprising any number of contact terms
}

\author{
H. Witała ${ }^{\text {a }}$ J. Golak, R. Skibiński \\ M. Smoluchowski Institute of Physics, Jagiellonian University, 30348 Kraków, Poland
}

Received: 6 May 2021 / Accepted: 8 July 2021

(C) The Author(s) 2021

Communicated by Vittorio Somà

\begin{abstract}
We demonstrate a computational scheme which drastically decreases the required time to get theoretical predictions based on chiral two- and three-nucleon forces for observables in three-nucleon continuum. For a three-nucleon force containing $\mathrm{N}$ short-range terms all workload is reduced to solving N+1 Faddeev-type integral equations. That done, computation of observables for any combination of strengths of the contact terms is done in a flash. We demonstrate on example of the elastic nucleon-deuteron scattering observables the high precision of the proposed emulator and its capability to reproduce exact results.
\end{abstract}

\section{Introduction}

Since the birth of nuclear physics the nuclear force problem has been at the centre of experimental and theoretical studies. Extensive efforts based on purely phenomenological approaches or incorporating the meson-exchange picture have led to numerous nucleon-nucleon (NN) potentials, able to describe a vast amount of available data [1] with high precision. A major breakthrough occurred with the emergence of the effective field theory (EFT) concept [2], which paved the way for developing precise nuclear forces [36]. The progress in constructing nuclear forces within the EFT approach is presently documented by the availability of numerous high precision NN potentials developed by the Bochum-Bonn [7, 8], Idaho-Salamanca [9], and Bochum [10] groups. These forces provide a very good description of the NN data set.

Applications of the EFT approach in the form of chiral perturbation theory (ChPT) have resulted not only in the theoretically well grounded NN potentials but also for the first

a e-mail: henryk.witala@uj.edu.pl (corresponding author) time have given a possibility to apply in practical calculations $\mathrm{NN}$ forces augmented by consistent $3 \mathrm{~N}$ interactions, derived within the same formalism. Understanding of nuclear spectra and reactions based on these consistent chiral two- and manybody forces has become a hot topic of present day few-body studies [11].

The first nonvanishing contributions to the $3 \mathrm{~N}$ force (3NF) appear at next-to-next-to-leading order of chiral expansion ( $\left.\mathrm{N}^{2} \mathrm{LO}\right)[3,12]$ and comprise in addition to the $2 \pi$-exchange term two contact contributions with strength parameters $c_{D}$ and $c_{E}$ [13]. The chiral $3 \mathrm{NF}$ at $\mathrm{N}^{3} \mathrm{LO}$ was derived in $[14,15]$. At that order three components of long-range character [14] supplemented by the short-range terms [15] contribute to $3 \mathrm{NF}$. The $3 \mathrm{NF}$ at $\mathrm{N}^{3} \mathrm{LO}$ order does not involve any new unknown low-energy constants and depends only on two parameters, $c_{D}$ and $c_{E}$, that parameterize the leading one-pion-contact term and the $3 \mathrm{~N}$ contact term present already at $\mathrm{N}^{2} \mathrm{LO}$. The $c_{D}$ and $c_{E}$ values need to be then fixed at this order, as at $\mathrm{N}^{2} \mathrm{LO}$, from a fit to few-nucleon data. At the higher order, $\mathrm{N}^{4} \mathrm{LO}$, in addition to long- and intermediate-range interactions generated by pion-exchange diagrams $[16,17]$, the chiral $\mathrm{N}^{4} \mathrm{LO} 3 \mathrm{NF}$ involves thirteen purely short-range operators, which have been worked out in $[18,19]$.

Since the advent of numerically exact three-nucleon continuum Faddeev calculations the elastic nucleon-deuteron (Nd) scattering and the deuteron breakup reaction have been a powerful tool to test modern models of the nuclear forces [20-22] and the question about the importance of 3NF has developed into the main topic of $3 \mathrm{~N}$ system studies. That issue has been given a new impetus by the ChPT-based achievements and the possibility to apply consistent two- and many-body nuclear forces, derived within this framework, in $3 \mathrm{~N}$ continuum calculations. 
Using chiral $3 \mathrm{NF}$ in $3 \mathrm{~N}$ continuum requires numerous time consuming computations with varying strengths of the contact terms in order to establish their values. They can be determined for example from the ${ }^{3} \mathrm{H}$ binding energy and the minimum of the elastic Nd scattering differential cross section at the energy $\left(E_{l a b} \approx 70 \mathrm{MeV}\right)$, where the effects of $3 \mathrm{NF}$ start to emerge in elastic Nd scattering [23,24]. Specifically at $\mathrm{N}^{2} \mathrm{LO}$, after establishing the so-called $\left(c_{D}, c_{E}\right)$ correlation line, which for a particular chiral NN potential combined with a $\mathrm{N}^{2} \mathrm{LO} 3 \mathrm{NF}$ gives pairs of $\left(c_{D}, c_{E}\right)$ values reproducing the ${ }^{3} \mathrm{H}$ binding energy, a fit to experimental data for the elastic $\mathrm{Nd}$ cross section is performed to determine the $c_{D}$ and $c_{E}$ strengths. Fine-tuning of the $3 \mathrm{~N}$ Hamiltonian parameters requires an extensive analysis of available $3 \mathrm{~N}$ elastic $\mathrm{Nd}$ scattering and breakup data. That ambitious goal calls for a significant reduction of computer time necessary to solve the $3 \mathrm{~N}$ Faddeev equations and to calculate the observables. Thus finding an efficient emulator for exact solutions of the $3 \mathrm{~N}$ Faddeev equations seems to be essential and of high priority.

In Ref. [25] we proposed such an emulator which enables us to reduce significantly the required time of calculations. We tested its efficiency as well as ability to accurately reproduce exact solutions of $3 \mathrm{~N}$ Faddeev equations. In the present study we introduce a new computational scheme, based on the perturbative approach of [25], which even by far more reduces the computer time required to obtain the observables in the elastic nucleon-deuteron scattering and deuteron breakup reactions at any energy, and which is well-suited for calculations with varying strengths of the contact terms in a chiral 3NF.

\section{The new emulator}

Before presenting this new emulator, for the reader's convenience we shortly outline the main points of the $3 \mathrm{~N}$ Faddeev formalism and of the perturbative treatment of Ref. [25]. For details of the formalism and numerical performance we refer to $[20,26-28]$.

Neutron-deuteron (nd) scattering with nucleons interacting via NN interactions $v_{N N}$ and a $3 \mathrm{NF} V_{123}=V^{(1)}+V^{(2)}+$ $V^{(3)}$, is described in terms of a breakup operator $T$ satisfying the Faddeev-type integral equation [20,26,27]

$$
\begin{aligned}
T|\phi\rangle= & t P|\phi\rangle+\left(1+t G_{0}\right) V^{(1)}(1+P)|\phi\rangle+t P G_{0} T|\phi\rangle \\
& +\left(1+t G_{0}\right) V^{(1)}(1+P) G_{0} T|\phi\rangle .
\end{aligned}
$$

The $2 \mathrm{~N} t$-matrix $t$ is the solution of the Lippmann-Schwinger equation with the interaction $v_{N N} . V^{(1)}$ is the part of a $3 \mathrm{NF}$ which is symmetric under the interchange of nucleons 2 and 3: $V_{123}=V^{(1)}(1+P)$. The permutation operator $P=P_{12} P_{23}+P_{13} P_{23}$ is given in terms of the transposi- tion operators, $P_{i j}$, which interchange nucleons $i$ and $j$. The initial state $|\phi\rangle=\left|\mathbf{q}_{0}\right\rangle\left|\phi_{d}\right\rangle$ describes the free motion of the neutron and the deuteron with the relative momentum $\mathbf{q}_{0}$ and contains the internal deuteron wave function $\left|\phi_{d}\right\rangle . G_{0}$ is the free three-body resolvent. The amplitude for elastic scattering leading to the final nd state $\left|\phi^{\prime}\right\rangle$ is then given by [20,27]

$$
\begin{aligned}
\left\langle\phi^{\prime}|U| \phi\right\rangle= & \left\langle\phi^{\prime}\left|P G_{0}^{-1}\right| \phi\right\rangle+\left\langle\phi^{\prime}\left|V^{(1)}(1+P)\right| \phi\right\rangle \\
& +\left\langle\phi^{\prime}\left|V^{(1)}(1+P) G_{0} T\right| \phi\right\rangle+\left\langle\phi^{\prime}|P T| \phi\right\rangle,
\end{aligned}
$$

while the amplitude for the breakup reaction reads

$\left\langle\mathbf{p q}\left|U_{0}\right| \phi\right\rangle=\langle\mathbf{p q}|(1+P) T| \phi\rangle$,

where the free breakup channel state $|\mathbf{p q}\rangle$ is defined in terms of the Jacobi (relative) momenta $\mathbf{p}$ and $\mathbf{q}$.

We solve Eq. (1) in the momentum-space partial-wave basis $|p q \tilde{\alpha}\rangle$, determined by the magnitudes of the Jacobi momenta $p$ and $q$ and a set of discrete quantum numbers $\tilde{\alpha}$ comprising the $2 \mathrm{~N}$ subsystem spin, orbital and total angular momenta $s, l$ and $j$, as well as the spectator nucleon orbital and total angular momenta with respect to the center of mass (c.m.) of the $2 \mathrm{~N}$ subsystem, $\lambda$ and $I$ :

$|p q \tilde{\alpha}\rangle \equiv\left|p q(l s) j\left(\lambda \frac{1}{2}\right) I(j I) J\left(t \frac{1}{2}\right) T\right\rangle$.

The total $2 \mathrm{~N}$ and spectator angular momenta $j$ and $I$ as well as isospins $t$ and $\frac{1}{2}$, are finally coupled to the total angular momentum $J$ and isospin $T$ of the $3 \mathrm{~N}$ system. In practice a converged solution of Eq. (1) using partial wave decomposition in momentum space at a given energy $E$ requires taking all $3 \mathrm{~N}$ partial wave states up to the $2 \mathrm{~N}$ angular momentum $j_{\max }=5$ and the $3 \mathrm{~N}$ angular momentum $J_{\max }=\frac{25}{2}$, with the $3 \mathrm{~N}$ force acting up to the $3 \mathrm{~N}$ total angular momentum $J=7 / 2$. The number of resulting partial waves (equal to the number of coupled integral equations in two continuous variables $p$ and $q$ ) amounts to 142 . The required computer time to get one solution on a personal computer is about $\approx 2 \mathrm{~h}$. In the case when such calculations have to be performed for a big number of varying $3 \mathrm{NF}$ parameters, time restrictions become prohibitive. Fortunately, the perturbative approach of Ref. [25] leads to a significant reduction of the required computational time.

Let us consider a chiral $3 \mathrm{NF}$ at a given order of chiral expansion with variable strengths of its contact terms. The $3 \mathrm{NF}$ at $\mathrm{N}^{2} \mathrm{LO}$ has one parameter-free term $(2 \pi$-exchange contribution) and two short-range terms with strength parameters $c_{D}$ and $c_{E}$. At $\mathrm{N}^{3} \mathrm{LO}$ there are more contributing parameter-free parts but again only two contact terms. At $\mathrm{N}^{4}$ LO parameter-free contributions are supplemented by fifteen short-range terms with strengths: $c_{D}, c_{E}, c_{E_{1}}, \ldots, c_{E_{13}}$. All these contact terms are restricted to small $3 \mathrm{~N}$ total angular momenta and to only few partial wave states for a given total $3 \mathrm{~N}$ angular momentum $J$ and parity $\pi$. For example 
for $J^{\pi}=7 / 2^{ \pm}$all matrix elements $<p q \tilde{\alpha}\left|V^{(1)}\right| p^{\prime} q^{\prime} \tilde{\alpha}^{\prime}>$ proportional to $c_{E_{1}}$ and $c_{E_{7}}$ vanish, while the $c_{D}$ and $c_{E}$ terms are nonzero only for a restricted number of $\tilde{\alpha}, \tilde{\alpha}^{\prime}$ pairs (mostly these containing ${ }^{1} S_{0}$ and ${ }^{3} S_{1}-{ }^{3} D_{1}$ quantum numbers) $[12,13]$. Bearing that in mind and taking into account the fact that contact terms yield a small contribution to the $3 \mathrm{~N}$ potential energy compared to the leading, parameter-free part, it is possible to apply a perturbative approach in order to include the contact terms.

We split the $V^{(1)}$ part of a $3 \mathrm{NF}$ into a parameter-free term $V\left(\theta_{0}\right)$ and a sum of $N$ contact terms $c_{i} \Delta V_{i}$ with strengths $c_{i}$.

$V^{(1)}=V\left(\theta_{0}\right)+\Delta V(\theta)=V\left(\theta_{0}\right)+\sum_{i=1}^{N} c_{i} \Delta V_{i}$,

with $\theta_{0}=\left(c_{i}=0, i=1, \ldots, N\right)$ and $\theta=\left(c_{i}, i=\right.$ $1, \ldots, N)$ being the sets of contact terms strength values, for which we would like to find solution of Eq. (1).

We divide the $3 \mathrm{~N}$ partial wave states $\tilde{\alpha}$ into two sets: $\beta$ and the remaining one, $\alpha$. The $\beta$ set contains states with nonvanishing elements of $\Delta V(\theta)$ and states most strongly coupled to them through the permutation operator $P$. This set of states is sufficient for the convergence of $\Delta T(\theta)$ in the second equation (7), also in the intermediate states. Introducing $T\left(\theta_{0}\right)$ and $\Delta T(\theta)$ such that $T \equiv T(\theta)=T\left(\theta_{0}\right)+\Delta T(\theta)$, and using the fact, that $\Delta V(\theta)$ has nonvanishing elements only for channels $|\beta\rangle$, one gets from Eq. (1) (omitting the Jacobi momenta in notation of partial wave states) two separate equations for $\left\langle\alpha\left|T\left(\theta_{0}\right)\right| \phi\right\rangle$ and $\langle\alpha|\Delta T(\theta)| \phi\rangle[25]$ :

$$
\begin{aligned}
\left\langle\alpha\left|T\left(\theta_{0}\right)\right| \phi\right\rangle= & \langle\alpha|t P| \phi\rangle+\left\langle\alpha\left|\left(1+t G_{0}\right) V\left(\theta_{0}\right)(1+P)\right| \phi\right\rangle \\
& +\left\langle\alpha\left|t P G_{0} T\left(\theta_{0}\right)\right| \phi\right\rangle \\
& +\left\langle\alpha\left|\left(1+t G_{0}\right) V\left(\theta_{0}\right)(1+P) G_{0} T\left(\theta_{0}\right)\right| \phi\right\rangle \\
\langle\alpha|\Delta T(\theta)| \phi\rangle= & \left\langle\alpha\left|t P G_{0} \Delta T(\theta)\right| \phi\right\rangle \\
& +\left\langle\alpha\left|\left(1+t G_{0}\right) V\left(\theta_{0}\right)(1+P) G_{0} \Delta T(\theta)\right| \phi\right\rangle,
\end{aligned}
$$

as well as for $\left\langle\beta\left|T\left(\theta_{0}\right)\right| \phi\right\rangle$ and $\langle\beta|\Delta T(\theta)| \phi\rangle$ :

$$
\begin{aligned}
\left\langle\beta\left|T\left(\theta_{0}\right)\right| \phi\right\rangle= & \langle\beta|t P| \phi\rangle+\left\langle\beta\left|\left(1+t G_{0}\right) V\left(\theta_{0}\right)(1+P)\right| \phi\right\rangle \\
& +\left\langle\beta\left|t P G_{0} T\left(\theta_{0}\right)\right| \phi\right\rangle \\
& +\left\langle\beta\left|\left(1+t G_{0}\right) V\left(\theta_{0}\right)(1+P) G_{0} T\left(\theta_{0}\right)\right| \phi\right\rangle \\
\langle\beta|\Delta T(\theta)| \phi\rangle= & \left\langle\beta\left|\left(1+t G_{0}\right) \Delta V(\theta)(1+P)\right| \phi\right\rangle \\
& +\left\langle\beta\left|\left(1+t G_{0}\right) \Delta V(\theta)(1+P) G_{0} T\left(\theta_{0}\right)\right| \phi\right\rangle \\
& +\left\langle\beta\left|\left(1+t G_{0}\right) V\left(\theta_{0}\right)(1+P) G_{0} \Delta T(\theta)\right| \phi\right\rangle \\
& +\left\langle\beta\left|\left(1+t G_{0}\right) \Delta V(\theta)(1+P) G_{0} \Delta T(\theta)\right| \phi\right\rangle \\
& +\left\langle\beta\left|t P G_{0} \Delta T(\theta)\right| \phi\right\rangle .
\end{aligned}
$$

The first equations in (6) and (7) are the Faddeev equations (1) for $T\left(\theta_{0}\right)$. The second equation in the set (7) for
$\langle\beta|\Delta T(\theta)| \phi\rangle$ can be solved within the set of channels $|\beta\rangle$ only. Using this solution, $\langle\alpha|\Delta T(\theta)| \phi\rangle$ is then computed by:

$$
\begin{aligned}
\langle\alpha|\Delta T(\theta)| \phi\rangle= & \left\langle\alpha\left|t P G_{0} \sum_{\beta} \int_{p^{\prime} q^{\prime}}\right| p^{\prime} q^{\prime} \beta\right\rangle\left\langle p^{\prime} q^{\prime} \beta|\Delta T(\theta)| \phi\right\rangle \\
& +\langle\alpha|\left(1+t G_{0}\right) V\left(\theta_{0}\right)(1+P) G_{0} \\
& \sum_{\beta} \int_{p^{\prime} q^{\prime}}\left|p^{\prime} q^{\prime} \beta\right\rangle\left\langle p^{\prime} q^{\prime} \beta|\Delta T(\theta)| \phi\right\rangle,
\end{aligned}
$$

and finally, $\langle\tilde{\alpha}|T(\theta)| \phi\rangle$ is calculated.

The outlined above procedure constitutes the perturbative approach of Ref. [25]. In short, one solves the $3 \mathrm{~N}$ Faddeev equation (1) exactly with the NN potential combined with the $3 \mathrm{NF}$ restricted to the parameter free term (set $\left.\theta_{0}=(0, \ldots, 0)\right)$. The solution with the $\theta_{0}$ set forms a starting point in the perturbative treatment of Eqs. (6-8) and has to be calculated only once, regardless of how many variations of strength parameters are required. In the next step, the proper perturbative treatment is performed, solving first the second equation in set (7). Having determined $\langle\alpha|\Delta T(\theta)| \phi\rangle$ from Eq.(8) the emulator solution $\langle\tilde{\alpha}|T(\theta)| \phi\rangle$ is calculated (in the following this emulator will be denoted by $E \Delta T)$. That allows one to reduce the required computation time and to reproduce surprisingly well the exact predictions for neutron-deuteron (nd) elastic scattering as well as for nd breakup observables [25]. To be specific, taking set $|\beta\rangle$ which includes all $2 \mathrm{~N}$ states with the total $2 \mathrm{~N}$ angular momenta $j \leq 2$, leads to a reduction of the computing time by a factor of approximately 4 in comparison to the exact calculations. Note that it takes approximately 30 minutes on a personal computer to solve Eq. (1), provided that the $V\left(\theta_{0}\right)(1+P)$ and $V\left(\theta_{i}\right)(1+P)$ kernels, acting in $\left(1+t G_{0}\right) V(\theta)(1+P) G_{0} T(\theta)|\phi\rangle$ term of Eq. (1), are prepared in advance, with the strengths $\theta_{i}=\left(c_{i}=1, c_{k \neq i}=0\right)$.

In spite of such a large reduction, the computational time can be even further decreased and calculation of $3 \mathrm{~N}$ continuum observables made in a flash. This notion is based on the observation that among three kernel-terms in the second equation of set (7), it is possible (because of the smallness of $\Delta V(\theta))$ to neglect the term $\langle\beta|(1+$ $\left.t G_{0}\right) \Delta V(\theta)(1+P) G_{0} \Delta T(\theta)|\phi\rangle$. The resulting integral equation for $\langle\beta|\Delta T(\theta)| \phi\rangle$ :

$$
\begin{aligned}
\langle\beta|\Delta T(\theta)| \phi\rangle= & \left\langle\beta\left|\left(1+t G_{0}\right) \Delta V(\theta)(1+P)\right| \phi\right\rangle \\
& +\left\langle\beta\left|\left(1+t G_{0}\right) \Delta V(\theta)(1+P) G_{0} T\left(\theta_{0}\right)\right| \phi\right\rangle \\
& +\left\langle\beta\left|\left(1+t G_{0}\right) V\left(\theta_{0}\right)(1+P) G_{0} \Delta T(\theta)\right| \phi\right\rangle \\
& +\left\langle\beta\left|t P G_{0} \Delta T(\theta)\right| \phi\right\rangle,
\end{aligned}
$$

permits one to transfer the linear dependence on the strengths $c_{i}$ from the $\Delta V(\theta)$ on the $\Delta T(\theta)$. Namely, let $\left\langle\beta\left|\Delta T_{i}\right| \phi\right\rangle$ be a solution of Eq. (9) for a set $\theta_{i}=\left(c_{i}=1, c_{k \neq i}=0\right)$ :

$\left\langle\beta\left|\Delta T_{i}\right| \phi\right\rangle \equiv\left\langle\beta\left|\left(1+t G_{0}\right) \Delta V_{i}(1+P)\right| \phi\right\rangle$ 


$$
\begin{aligned}
& +\left\langle\beta\left|\left(1+t G_{0}\right) \Delta V_{i}(1+P) G_{0} T\left(\theta_{0}\right)\right| \phi\right\rangle \\
& +\left\langle\beta\left|\left(1+t G_{0}\right) V\left(\theta_{0}\right)(1+P) G_{0} \Delta T_{i}\right| \phi\right\rangle \\
& +\left\langle\beta\left|t P G_{0} \Delta T_{i}\right| \phi\right\rangle .
\end{aligned}
$$

Multiplying (10) by $c_{i}$ and summing over $i$ one gets:

$$
\begin{aligned}
\langle\beta| & \sum_{i} c_{i} \Delta T_{i}|\phi\rangle \\
\equiv & \left\langle\beta\left|\left(1+t G_{0}\right) \sum_{i} c_{i} \Delta V_{i}(1+P)\right| \phi\right\rangle \\
& +\left\langle\beta\left|\left(1+t G_{0}\right) \sum_{i} c_{i} \Delta V_{i}(1+P) G_{0} T\left(\theta_{0}\right)\right| \phi\right\rangle \\
& +\left\langle\beta\left|\left(1+t G_{0}\right) V\left(\theta_{0}\right)(1+P) G_{0} \sum_{i} c_{i} \Delta T_{i}\right| \phi\right\rangle \\
& +\left\langle\beta\left|t P G_{0} \sum_{i} c_{i} \Delta T_{i}\right| \phi\right\rangle,
\end{aligned}
$$

and the solution of Eq. (9) is given by:

$$
\langle\beta|\Delta T(\theta)| \phi\rangle=\sum_{i=1}^{N} c_{i}\left\langle\beta\left|\Delta T_{i}\right| \phi\right\rangle .
$$

In this way at a given energy the computation of observables in the elastic Nd scattering and deuteron breakup reaction for any combination of strengths $c_{i}$ of contact terms is reduced to solving once $N+1$ Faddeev equations: one equation for $T\left(\theta_{0}\right)$ and $\mathrm{N}$ equations for $\Delta T_{i}$. In the first step, solution for $\left\langle\alpha(\beta)\left|T\left(\theta_{0}\right)\right| \phi\right\rangle$ is found. Then Eq. (10) is solved for $\left\langle\beta\left|\Delta T_{i}\right| \phi\right\rangle$, from which the $\left\langle\alpha\left|\Delta T_{i}\right| \phi\right\rangle$ is calculated using Eq. (8). The above computations need to be done only once and then for any combination of the strengths $c_{i}$ the amplitude $\left\langle\tilde{\alpha}\left|T\left(\theta=\left(c_{i}, i=1, \ldots, N\right)\right)\right| \phi\right\rangle$ is obtained by trivial summation.

For a calculation of elastic scattering observables the required sum of the second and the third term in Eq. (2) is obtained by:

$$
\begin{aligned}
\langle\beta| & V^{(1)}(\theta)(1+P)|\phi\rangle+\left\langle\beta\left|V^{(1)}(\theta)(1+P) G_{0} T(\theta)\right| \phi\right\rangle \\
= & \left\langle\beta\left|V\left(\theta_{0}\right)(1+P)\right| \phi\right\rangle+\left\langle\beta\left|V\left(\theta_{0}\right)(1+P) G_{0} T\left(\theta_{0}\right)\right| \phi\right\rangle \\
& +\sum_{i} c_{i}\left[\left\langle\beta\left|\Delta V_{i}(1+P)\right| \phi\right\rangle\right. \\
& +\left\langle\beta\left|\Delta V_{i}(1+P) G_{0} T\left(\theta_{0}\right)\right| \phi\right\rangle \\
& \left.+\left\langle\beta\left|V\left(\theta_{0}\right)(1+P) G_{0} \Delta T_{i}\right| \phi\right\rangle\right] \\
& +\sum_{i, k} c_{i} c_{k}\left\langle\beta\left|\Delta V_{i}(1+P) G_{0} \Delta T_{k}\right| \phi\right\rangle .
\end{aligned}
$$

It is interesting to note that while the breakup amplitude (3) is linear in $3 \mathrm{~N}$ contact strengths, the elastic scattering amplitude contains, as a consequence of the third term present in Eq. (2), also terms quadratic in these strengths.

\section{Results and discussion}

The above computational scheme forms the new emulator, which will be denoted in the following by $E \Delta T_{i}$. To check its quality and efficiency as well as to compare it with $E \Delta T$ we have chosen, as in Ref. [25], the SMS ${ }^{4} \mathrm{LO}^{+}$chiral potential of the Bochum group [10], with the regularization cutoff $\Lambda=450 \mathrm{MeV}$, and combined it with the chiral $\mathrm{N}^{2} \mathrm{LO}$ $3 \mathrm{NF}$. We solved the $3 \mathrm{~N}$ Faddeev equation (1) exactly at two incoming neutron energies $E=70$ and $190 \mathrm{MeV}$ with that combination as well as with this NN potential supplemented with the parameter free $2 \pi$-exchange term of the $\mathrm{N}^{2} \mathrm{LO} 3 \mathrm{NF}$ (set $\left.\theta_{0}=\left(c_{D}=0, c_{E}=0\right)\right)$. The first energy was taken from a region where $3 \mathrm{NF}$ effects start to appear in $3 \mathrm{~N}$ continuum observables and the second one from a range with well-developed $3 \mathrm{NF}$ effects $[23,24]$. The solution with the $\theta_{0}$ set together with solutions $\left\langle\beta\left|\Delta T_{i}\right| \phi\right\rangle$ of $\mathrm{N}$ integral equations (10) with the set of $3 \mathrm{~N}$ channels $|\beta\rangle$, comprising all $2 \mathrm{~N}$ states with the total angular momentum $j \leq 2$, form a starting point in the proposed computational scheme and have to be calculated only once. Also $\left\langle\alpha\left|\Delta T_{i}(\theta)\right| \phi\right\rangle$ is computed only once according to Eq. (8), and, in parallel with those calculations, all terms in Eq. (13) independent from $c_{i}$. Based on these quantities we calculated the emulator solution $\langle\tilde{\alpha}|T(\theta)| \phi\rangle$. Our new scheme performed for $N=2$ leads to reduction by a factor of $\approx 50$ of the computer time required by the perturbative approach of Ref. [25]. Computation of all elastic scattering observables or observables in one exclusive breakup geometry for a particular set of strengths $\theta=\left(c_{i}, i=1, \ldots, N\right)$, in the perturbative approach requires $\approx 8$ minutes of a personal computer time, while the new scheme requires only $\approx 10$ seconds. Since the calculation of $\langle\tilde{\alpha}|T(\theta)| \phi\rangle$ from $\left\langle\tilde{\alpha}\left|T\left(\theta_{0}\right)\right| \phi\right\rangle$ and $\left\langle\tilde{\alpha}\left|\Delta T_{i}(\theta)\right| \phi\right\rangle$ takes practically no time, the drastic reduction of time in the new scheme is independent from the number of the contact terms $N$.

The emulators $E \Delta T_{i}$ and $E \Delta T$ are approximations of the exact results. To estimate the quality of these approximations we show in Fig. 1 at both energies the distributions of percentage deviations from exact results $O_{\text {exact }}$ predictions of both emulators $O_{\text {appr }}: \Delta=\left(O_{\text {appr }}-O_{\text {exact }}\right) / O_{\text {exact }} \times$ 100.0 , for all nonvanishing nd elastic scattering observables $O$ (cross section, nucleon and deuteron analyzing powers as well as spin correlation and spin transfer coefficients between all participating particles, altogether 51 observables). They were calculated on a uniform grid of 73 c.m. angles $\theta_{c . m .} \in\left(0^{\circ}, 180^{\circ}\right)$. These distributions encompass six sets of strengths values $\left(c_{D}, c_{E}\right)=(1,1),(2,1),(4,1)$, $(6,1),(8,1)$, and $(10,1)$ (according to the notation of Refs. $[12,13])$. The precision of both emulators at both energies is similar and amounts to $\approx 1-2 \%$, with $E \Delta T$ being slightly more precise than $E \Delta T_{i}$.

To demonstrate the power of the new emulator we show in Fig. 2 the color map of $\chi^{2}$ values in the plane of strengths 



Fig. 1 Histograms of percentage deviations from exact results of the predictions by the emulator $E \Delta T$ (red lines) or $E \Delta T_{i}$ (blue lines), for 51 nonvanishing observables of the elastic nd scattering and 6 sets of $\left(c_{D}, c_{E}\right)$ strengths. For details see text

$\left(c_{D}, c_{E}\right)$ for elastic scattering cross section at $E=70 \mathrm{MeV}$. That map was obtained with about 5000 values of strength combinations, both varied in step of 0.1 . The minimum of $\chi^{2}$ valley is indicated by black squares. It crosses with $\chi^{2}$ values for the ${ }^{3} \mathrm{H}$ correlation line, shown by yellow dots, in their minimum. The resulting values of strengths and their errors are $c_{D}=2.910 \pm 0.140$ and $c_{E}=0.385 \pm 0.015$. Using these strengths we demonstrate in Fig. 3 importance of contributions to the cross section from individual terms of the $\mathrm{N}^{2} \mathrm{LO} 3 \mathrm{NF}$. We show percentage changes in the cross section values calculated solely with the NN potential by adding the parameter-free term itself, and by the combination of that term with the D- or E-terms. The $2 \pi$-exchange contribution as well as the short-range D-term are important at both energies. The contribution of the $\mathrm{E}$ term is negligible at $E=70 \mathrm{MeV}$ but clearly visible at $190 \mathrm{MeV}$.

\section{Summary and conclusions}

In summary, we presented a new powerful calculational scheme which enables us to take efficiently into account any number of contact terms of a chiral $3 \mathrm{NF}$ in the $3 \mathrm{~N}$ continuum Faddeev calculations. That approach facilitates a reduction of the time required to compute observables for a given set of strengths to seconds and is thus especially suited to repeated calculations with varying strengths of contact terms. We demonstrated that the proposed emulator reproduces very well the exact predictions for $3 \mathrm{~N}$ continuum observables. It is conceivable that with the help of the constructed emulator of the exact solutions of the $3 \mathrm{~N}$ Faddeev equation fine tuning of a $3 \mathrm{~N}$ Hamiltonian parameters based on available $3 \mathrm{~N}$ scattering data is feasible. To that aim not only existing neutron-

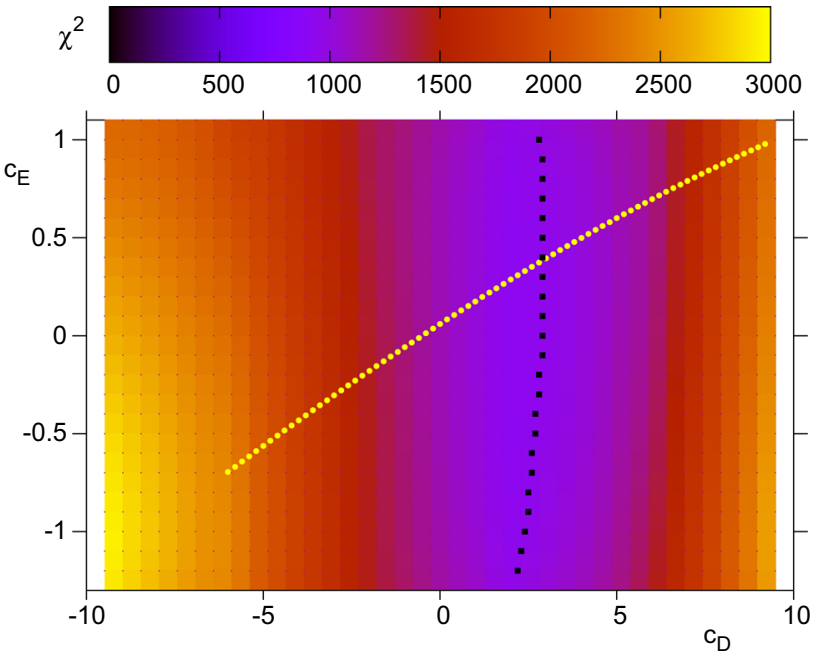

Fig. 2 The color map of $\chi^{2}$ values for nd elastic scattering cross section at $E=70 \mathrm{MeV}$, in the plane of strengths $\left(c_{D}, c_{E}\right)$ of $\mathrm{N}^{2} \mathrm{LO} 3 \mathrm{NF}$ contact terms. It was obtained with the $E \Delta T_{i}$ emulator for combination of chiral $\mathrm{NN} \mathrm{N}^{4} \mathrm{LO}^{+}$potential and $\mathrm{N}^{2} \mathrm{LO} 3 \mathrm{NF}$ and computed from about 5000 strengths combinations. The proton-deuteron cross section data were taken from [29] and $\chi^{2}$ calculated for c.m. angles in the range $\theta_{\text {c.m. }} \in\left(62.18^{\circ}, 158.33^{\circ}\right)$. The (black) squares show the position of the $\chi^{2}$-minimum at given $c_{E}$-value and (yellow) dots depict the ${ }^{3} \mathrm{H}$ correlation line



Fig. 3 The (red) solid lines show percentage changes of the nd elastic scattering cross section at $E=70$ and $190 \mathrm{MeV}$, predicted with SMS $\mathrm{N}^{4} \mathrm{LO}^{+} \mathrm{NN}$ potential, induced by $\mathrm{N}^{2} \mathrm{LO} 3 \mathrm{NF}$ with strengths of the contact terms $c_{D}=2.910$ and $c_{E}=0.385$. The (blue) dashed lines correspond to changes caused by a $3 \mathrm{NF}$ parameter-free term, while the (black) dash-dotted and (magenta) dash-double-dotted curves to changes by a $3 \mathrm{NF}$ parameter free $+\mathrm{D}$ and parameter free $+\mathrm{E}$ terms, respectively

deuteron elastic scattering and breakup data but also more abundant and precise proton-deuteron data should be used. However, before using the proton-deuteron data they should be corrected for the effects of the proton-proton Coulomb force acting in the proton-deuteron system. Such procedure involving estimation of the Coulomb corrections through the difference of calculations with and without Coulomb force included [21,30-32] have been already applied for example in [12]. 
Acknowledgements This study has been performed within Low Energy Nuclear Physics International Collaboration (LENPIC) project and was supported by the Polish National Science Center under Grant No. 2016/22/M/ST2/00173. The numerical calculations were performed on the supercomputer cluster of the JSC, Jülich, Germany.

Data Availability Statement This manuscript has no associated data or the data will not be deposited. [Authors' comment:All relevant data are already given in the figures.]

Open Access This article is licensed under a Creative Commons Attribution 4.0 International License, which permits use, sharing, adaptation, distribution and reproduction in any medium or format, as long as you give appropriate credit to the original author(s) and the source, provide a link to the Creative Commons licence, and indicate if changes were made. The images or other third party material in this article are included in the article's Creative Commons licence, unless indicated otherwise in a credit line to the material. If material is not included in the article's Creative Commons licence and your intended use is not permitted by statutory regulation or exceeds the permitted use, you will need to obtain permission directly from the copyright holder. To view a copy of this licence, visit http://creativecomm ons.org/licenses/by/4.0/.

\section{References}

1. R. Machleidt, Adv. Nucl. Phys. 19, 189 (1989)

2. S. Weinberg, Nucl. Phys. B 363, 3 (1991)

3. U. van Kolck, Phys. Rev. C 49, 2932 (1994)

4. E. Epelbaum, W. Glöckle, U.-G. Meißner, Nucl. Phys. A 747, 362 (2005)

5. E. Epelbaum, Prog. Part. Nucl. Phys. 57, 654 (2006)

6. R. Machleidt, D.R. Entem, Phys. Rep. 503, 1 (2011)

7. E. Epelbaum, H. Krebs, U.-G. Meißner, Eur. Phys. J. A 51, 53 (2015)

8. E. Epelbaum, H. Krebs, U.-G. Meißner, Phys. Rev. Lett. 115, $122301(2015)$

9. D.R. Entem, R. Machleidt, Y. Nosyk, Phys. Rev. C 96, 024004 (2017)
10. P. Reinert, H. Krebs, E. Epelbaum, Eur. Phys. J. A 54, 86 (2018)

11. E. Epelbaum, LENPIC Collaboration et al., Phys. Rev. C 99, 024313 (2019)

12. E. Epelbaum et al., Phys. Rev. C 66, 064001 (2002)

13. E. Epelbaum et al., Eur. Phys. J. A 56, (2020)

14. V. Bernard, E. Epelbaum, H. Krebs, U.-G. Meißner, Phys. Rev. C 77, 064004 (2008)

15. V. Bernard, E. Epelbaum, H. Krebs, U.-G. Meißner, Phys. Rev. C 84, 054001 (2011)

16. H. Krebs, A. Gasparyan, E. Epelbaum, Phys. Rev. C 85, 054006 (2012)

17. H. Krebs, A. Gasparyan, E. Epelbaum, Phys. Rev. C 87, 054007 (2013)

18. L. Girlanda, A. Kievsky, M. Viviani, Phys. Rev. C 84(1-8), 014001 (2011)

19. L. Girlanda, A. Kievsky, M. Viviani, Phys. Rev. C 102(E), 019903 (2020). arXiv:1102.4799v3 [nucl-th]

20. W. Glöckle, H. Witała, D. Hüber, H. Kamada, J. Golak, Phys. Rep. 274, 107 (1996)

21. A. Kievsky, M. Viviani, S. Rosati, Phys. Rev. C 52, R15 (1995)

22. A. Deltuva, K. Chmielewski, P.U. Sauer, Phys. Rev. C 67, 034001 (2003)

23. H. Witała et al., Phys. Rev. C 63, 024007 (2001)

24. H. Witała, W. Glöckle, D. Hüber, J. Golak, H. Kamada, Phys. Rev. Lett. 81, 1183 (1998)

25. H. Witała, J. Golak, R. Skibiński, K. Topolnicki, Few-Body Syst. 62, $23(2021)$

26. H. Witała, T. Cornelius, W. Glöckle, Few-Body Syst. 3, 123 (1988)

27. D. Hüber, H. Kamada, H. Witała, W. Glöckle, Acta Phys. Pol. B 28, 1677 (1997)

28. W. Glöckle, The Quantum Mechanical Few-Body Problem (Springer, Berlin, 1983)

29. K. Sekiguchi et al., Phys. Rev. C 65, 034003 (2002)

30. A. Kievsky, M. Viviani, S. Rosati, Phys. Rev. C 60, 034001 (1999)

31. A. Deltuva, A.C. Fonseca, P.U. Sauer, Phys. Rev. C 71, 054005 (2005)

32. A. Deltuva, A.C. Fonseca, P.U. Sauer, Phys. Rev. C 72, 054004 (2005) 